\title{
Publicación completa de trabajos presentados en reuniones de una sociedad científica latinoamericana
}

\author{
Full-text publication of abstracts presented at meetings of a Latin American \\ scientific society
}

\author{
Dra. Manuela Dicembrino ${ }^{a}$, Dra. Mariana Anderson ${ }^{a}$ Dra. Ana Gabriela Velya ${ }^{a}$ Dra. María Fabiana Ossorio ${ }^{a}$ \\ Dr. Fernando Ferrero ${ }^{a}$
}

\begin{abstract}
RESUMEN
Objetivo. Estimar la proporción de trabajos presentados en reuniones de la Sociedad Latinoamericana de Investigación Pediátrica que fueron publicados en forma completa, describir motivos deno publicación y evaluar el impacto dela financiación en la tasa de publicación.

Métodos. Se incluyeron trabajos presentados en reuniones de 2005-2009. Se contactó a los autores y se los invitó a participar de una encuesta sobre publicación del trabajo o motivos para no hacerlo.

Resultados. Sobre 325 trabajos presentados, pudo obtenerse información de $232(71,4 \%)$. El 58,6\% alcanzó publicación en forma completa $(136 / 232)$. Los estudios con financiación $(40,0 \%)$ tuvieron más posibilidades de publicarse (OR: 2,2; IC 95\%:1,2-3,9). "Falta de tiempo" fue el motivo más frecuente de no publicación (35/96).

Conclusión. El 58,6\% de los trabajos presentados en reuniones de la Sociedad Latinoamericana de Investigación Pediátrica alcanzaron la publicación en forma completa; la falta de tiempo fue la causa más frecuente para no publicarlos. Las investigaciones con financiación tuvieron más posibilidades de ser publicadas.

Palabras clave. bibliografía de medicina, publicaciones periódicas, informe de investigación.
\end{abstract}

http:/ /dx.doi.org/10.5546/aap.2014.532

\section{INTRODUCCIÓN}

El proceso de investigación solo puede darse por concluido cuando sus resultados son sometidos al escrutinio de la comunidad científica. La relevancia de la publicación en la difusión del conocimiento es un hecho incuestionable, como también lo es la obligación moral de hacer públicos los resultados de cualquier investigación que pueda redundar en un beneficio social. Más

a. Docencia e Investigación, Hospital General de Niños Pedro de Elizalde, Buenos Aires.

Correspondencia:

Dr. Fernando Ferrero: fferrero@intramed.net

Conflicto de intereses: Ninguno que declarar.

Recibido: 3-6-2014

Aceptado: 21-7-2014 aún, la falta de publicación de resultados de investigaciones válidas puede colaborar a que se establezca o incremente "sesgo de publicación". ${ }^{1}$

Las sociedades científicas son el ámbito adecuado para la presentación inicial de investigaciones. Sin embargo, muchos de los trabajos presentados en congresos no alcanzan luego la publicación. ${ }^{2}$ En general, no más del $50 \%$ de los resúmenes presentados en congresos médicos alcanzan la publicación como artículo completo. ${ }^{3}$

En América Latina, la Sociedad Latinoamericana de Investigación Pediátrica (SLAIP), desde hace 50 años, estimula la producción científica local, pero se desconoce la proporción de trabajos presentados en sus reuniones que alcanzaron la publicación completa.

El objetivo del presente trabajo es estimar la proporción de trabajos presentados en las reuniones anuales de la SLAIP que fueron posteriormente publicados en revistas científicas, describir los motivos más frecuentemente mencionados como responsables de no publicación y evaluar si la financiación influye en la tasa de publicación.

\section{MATERIAL Y MÉTODOS}

Estudio observacional que incluye todos los trabajos presentados en reuniones anuales de la SLAIP entre 2005 y 2009. En cada estudio, se identificó y contactó (por correo electrónico, teléfono o en persona) a un autor y se lo invitó a participar de una breve encuesta (inicialmente, se contactó al primer autor; de no obtener respuesta, al último autor; y, luego, a cualquier otro autor). La encuesta fue autoadministrada y cerrada (ver Anexo). Se remitió una encuesta por cada trabajo, independientemente de que un sujeto fuera autor de varios trabajos.

Se consideró como publicación válida a aquella efectuada en una publicación periódica científica reconocida con Número Internacional Normalizado de Publicaciones Seriadas (ISSN, 
por sus siglas en inglés; International Standard Serial Number). Se verificó la precisión de la información recogida sobre publicaciones y se la contrastó con las bases de datos Medline y Lilacs, o con cada publicación en particular cuando lo anterior no fuera posible.

Análisis: Los datos de las encuestas fueron registrados disociados adecuadamente de todo dato filiatorio. Las variables categóricas se expresaron como porcentajes con intervalos de confianza del 95\%. La asociación entre proporciones se evaluó por medio de la prueba de chi cuadrado. Se asumió un nivel de significación de $\mathrm{p}<0,05$.

Consideraciones éticas: La encuesta incluyó información para el consentimiento. El análisis de la información se disoció de datos filiatorios. Se solicitó y obtuvo autorización de los comités institucionales del Hospital General de Niños Pedro de Elizalde, al que pertenecen los investigadores. Estudio registrado en el Registro Público de Proyectos de Investigación MS-GCBA con el número 32/13.

\section{RESULTADOS}

En el período estudiado (2005-2009), se presentaron 325 trabajos, se pudo contactar a los

TABLA 1. Trabajos presentados en cinco reuniones de la Sociedad Latinoamericana de Investigación Pediátrica

\begin{tabular}{lcccc}
\hline Año & Reunión & País & Ciudad & Trabajos \\
\hline 2005 & XLIII & Brasil & Ribeirao Preto & 86 \\
2006 & XLIV & Argentina & Bariloche & 57 \\
2007 & XLV & Chile & La Serena & 56 \\
2008 & XLVI & Perú & Urubamba & 60 \\
2009 & XLVII & Paraguay & Asunción & 66 \\
\hline Total & & & & 325 \\
\hline
\end{tabular}

autores de $263(80,9 \%)$ y se obtuvo información sobre $232(71,4 \%)$ (Tabla 1$)$.

Los trabajos se originaban en 11 países (Tabla 2).

E1 58,6\% (IC95\%: 52-65) de los trabajos alcanzaron la publicación en forma completa $(136 / 232)$. Se verificó la veracidad de la referencia bibliográfica en todos los casos. Noventa trabajos aparecieron en publicaciones indexadas en Medline, 74 en Lilacs y 48 en ambas bases de datos (Tabla 2).

De los 232 trabajos analizados, $92(39,6 \%)$ refirieron haber recibido financiación, 78 de fuentes estatales y 14 de fuentes privadas. Chile fue el país con mayor proporción de trabajos financiados $(59,0 \%)$ (Tabla 3$)$.

Los estudios que recibieron cualquier tipo de financiación tuvieron más posibilidades de ser publicados que aquellos que no la recibieron (69,5\% vs. 51,4\%; $\mathrm{p}=0,006$; OR: 2,1 IC95\%: 1,2-3,7).

De los 96 trabajos no publicados, se obtuvo información sobre el motivo en 66; la falta de tiempo fue el más frecuente $(\mathrm{n}=35)$ (Tabla 4$)$.

\section{DISCUSIÓN}

Nuestro estudio muestra que casi $60 \%$ de los trabajos presentados en reuniones de la SLAIP fueron publicados posteriormente de forma completa en revistas científicas.

Aunque la proporción de trabajos presentados en eventos científicos que alcanzan la publicación es muy variable, una revisión del tema que combina datos de 79 reportes (29729 resúmenes) calculó una tasa promedio de publicación de $44,5 \% .^{3}$

Esta tasa puede ser más elevada en algunos eventos desarrollados en el hemisferio norte, especialmente referidos a ciertas especialidades. En pediatría, las tasas de publicación oscilan entre $45 \%$ y $60 \%{ }^{4,5}$

TABla 2. Trabajos presentados en cinco reuniones de la Sociedad Latinoamericana de Investigación Pediátrica y aquellos que alcanzaron la publicación en texto completo, según país e indexación

\begin{tabular}{lcccccccccccc}
\hline País & Presentados & $\%$ & Analizados & $\%$ & Publicados & $\%$ & Medline & $\%$ & Lilacs & $\%$ & Ambas & $\%$ \\
\hline Argentina & 92 & 28,3 & 88 & 37,9 & 56 & 63,3 & 38 & 66,7 & 36 & 63,2 & 25 & 52,1 \\
Bolivia & 4 & 1,2 & 2 & 0,8 & 1 & 50,0 & 1 & 100,0 & 0 & 0,0 & 0 & 0,0 \\
Brasil & 74 & 22,8 & 36 & 15,5 & 24 & 66,7 & 19 & 79,2 & 18 & 75,0 & 14 & 17,6 \\
Chile & 79 & 24,3 & 56 & 24,1 & 37 & 66,1 & 30 & 81,1 & 13 & 35,1 & 8 & 16,7 \\
México & 10 & 3,1 & 8 & 3,4 & 4 & 50,0 & 0 & 0,0 & 0 & 0,0 & 0 & 0,0 \\
Perú & 43 & 13,2 & 32 & 13,8 & 9 & 28,1 & 3 & 33,3 & 6 & 66,7 & 1 & 2,1 \\
Paraguay & 14 & 4,3 & 8 & 3,4 & 3 & 37,5 & 0 & 0,0 & 1 & 33,3 & 0 & 0,0 \\
Otros & 9 & 2,7 & 2 & 0,9 & 2 & 100 & 0 & 0,0 & 0 & 0,0 & 0 & 0,0 \\
\hline \multicolumn{1}{r}{} & 325 & & 232 & & 136 & 58,6 & 90 & 66,1 & 74 & 54,4 & 48 & 35,2 \\
\hline
\end{tabular}


Aunque en Latinoamérica existe poca información sobre el tema, las tasas de publicación parecerían ser sensiblemente menores: se observa $25,5 \%$ en Chile (gastroenterología), ${ }^{6}$ 11,3\% en Argentina (pediatría) ${ }^{7}$ y $26,6 \%$ en Brasil (ortopedia). ${ }^{8}$

Es probable que la diferencia observada con otros eventos de la región esté relacionada con el perfil de la SLAIP y los asistentes a sus reuniones. Por un lado, en estas reuniones, no se aceptan reportes de casos, series de casos ni reportes sobre experiencias profesionales. Por otro lado, en general, los asistentes a estas reuniones se encuentran decididamente volcados a la investigación. Es sabido que aquellos que se dedican a la investigación reconocen la publicación de sus investigaciones como la meta final.

Encontramos que solo el $40 \%$ de las investigaciones referían haber recibido algún tipo de financiamiento. Con ligeras variantes por país, esta cifra muestra una realidad regional en la que la investigación cuenta con limitado apoyo; el gasto en innovación y desarrollo (2000-2005) para los países de la región oscila entre 0,1 (Perú) y $0,6 \%$ del PBI (Chile), ${ }^{9}$ en comparación con 1,13 de España. ${ }^{10}$ Cabe destacar que la mayor parte de esa inversión es realizada por los Gobiernos, desde $72 \%$ en Argentina a 53\% en Brasil, a diferencia de Estados Unidos, donde el aporte del Gobierno solo alcanza $31 \%{ }^{11}$

A pesar de todo, queda claro que el compromiso que implica el financiamiento recibido incrementa la posibilidad de que los resultados de la investigación alcancen la publicación en forma completa. ${ }^{12}$

Tabla 3. Financiamiento de las investigaciones presentadas en reuniones de la Sociedad Latinoamericana de Investigación Pediátrica (2005-2009), según país y origen del financiamiento

\begin{tabular}{lccccc}
\hline & Total & $\begin{array}{c}\text { Con } \\
\text { financiamiento }\end{array}$ & $\%$ & Público & Privado \\
\hline Argentina & 88 & 35 & 39,3 & 28 & 7 \\
Bolivia & 2 & 1 & 50,0 & 0 & 1 \\
Brasil & 36 & 13 & 36,2 & 12 & 1 \\
Chile & 56 & 33 & 59,0 & 31 & 2 \\
México & 8 & 1 & 12,5 & 0 & 1 \\
Paraguay & 8 & 2 & 25,0 & 1 & 1 \\
Perú & 32 & 7 & 21,9 & 6 & 1 \\
Venezuela & 2 & 0 & 0,0 & 0 & 0 \\
\hline Total & 232 & 92 & 39,6 & 78 & 14 \\
\hline
\end{tabular}

Aunque nuestro estudio solo incluye trabajos presentados en 5 reuniones de la SLAIP, esa cantidad de reuniones permite contar con un número suficiente de trabajos para estimar la tasa con razonable precisión, al mismo tiempo que el período elegido permite que los entrevistados recuerden fácilmente los trabajos involucrados y que estos hayan tenido tiempo de alcanzar la publicación, considerando que el tiempo promedio requerido suele ser no inferior a 2 años. ${ }^{13}$

Aunque no se analizaron los datos de los trabajos de los que no se recibió respuesta del autor, la elevada tasa de respuesta hace poco probable que exista un sesgo significativo. Tampoco se analizó la calidad de los trabajos; solo se consideró la publicación en revistas indexadas en Pubmed y Lilacs como un indicador indirecto de esta cualidad.

Además, otros estudios sobre el tema se basaron en la búsqueda en Pubmed de resúmenes presentados en eventos científicos, por lo que se pudo subestimar la tasa de publicación al considerar una sola fuente de datos. Nosotros preferimos recurrir a los autores y luego verificar en diferentes bases de datos la exactitud de la información recibida.

Si bien existe evidencia de que la situación en investigación biomédica ha mejorado significativamente en la región en los últimos 15 años, ${ }^{9}$ nuestro trabajo no permite hacer ninguna extrapolación a la situación regional dado que se limita a una especialidad en particular y participación mayoritaria de Argentina, Brasil y Chile en la sociedad científica evaluada.

En nuestro trabajo, la falta de tiempo fue

TABla 4. Motivos mencionados como responsables de no publicación en texto completo de los trabajos presentados en reuniones científicas

\begin{tabular}{lc}
\hline Motivo & n \\
\hline Falta de tiempo & 35 \\
No se alcanzó el tamaño muestral & 5 \\
Falta de acuerdo entre coautores & 4 \\
Pesimismo sobre la publicación & 3 \\
Rechazo & 3 \\
Resultados considerados no relevantes & 2 \\
Falta de interés & 1 \\
Trabajos con resultados similares & 1 \\
Otros & 12 \\
\hline Total & 66 \\
\hline
\end{tabular}


referida como principal causa de no publicación $(53 \%)$. Aun en un tema tan sensible como el de los estudios clínicos controlados de magnitud en oncología, la falta de tiempo es referida como principal causa para no publicar las investigaciones. ${ }^{14}$ Esto remarca la importancia de considerar este aspecto durante el planeamiento de la investigación, que incluye en su cronograma el tiempo que requiera la redacción del manuscrito y su manejo editorial. La falta de publicación es una constante preocupación en el mundo académico; inclusive se han desarrollado estrategias para intentar incrementarla, pero nada reemplaza al impulso y compromiso personal. ${ }^{15}$

En conclusión, encontramos que 58,6\% de los trabajos presentados en reuniones de la Sociedad Latinoamericana de Investigación Pediátrica alcanzaron la publicación en forma completa. Los estudios que contaban con algún tipo de financiación tuvieron más posibilidades de ser publicados. La falta de tiempo fue el motivo más frecuentemente referido como causa de no publicación.

\section{Agradecimiento}

Al Dr. Carlos Castillo Durán por la revisión crítica del manuscrito y su constante dedicación al progreso de la investigación pediátrica.

\section{REFERENCIAS}

1. Wager E, Williams P. Project Overcome failure to Publish Negative Findings Consortium. "Hardly worth the effort"? Medical journals' policies and their editors' and publishers' views on trial registration and publication bias: quantitative and qualitative study. BMJ 2013;347:f5248.

2. Von Elm E, Costanza MC, Walder B, Tramèr MR. More insight into the fate of biomedical meeting abstracts: a systematic review. BMC Med Res Methodol 2003;3:12.

3. Scherer RW, Langenberg P, Von Elm E. Full publication of results initially presented in abstracts. Cochrane Database Syst Rev 2007;(2):MR000005.
4. Carroll AE, Sox CM, Tarini BA, Ringold S, et al. Does presentation format at the Pediatric Academic Societies' annual meeting predict subsequent publication? Pediatrics 2003;112(6 Pt 1):1238-41.

5. Riordan FA. Do presenters to paediatric meetings get their work published? Arch Dis Child 2000;83(6):524-6.

6. Weitz JC, Silva H. Presentación versus publicación: análisis de trabajos presentados en Congresos Chilenos de Gastroenterología 1998 al 2002. Gastroenterol Latinoam 2005;16(3):287-92.

7. Canosa D, Ferrero F, Melamud A, Otero P, et al. Publicación completa de trabajos presentados en el $33^{\circ}$ Congreso Argentino dePediatria y análisis de factores que impidieron su publicación. Arch Argent Pediatr 2011;109(1):56-9.

8. Ejnisman L, Gomes GS, de Oliveira RG, Malavolta EA, et al. Publication rates of papers presented at the Brazilian Orthopedic Meeting. Acta Ortop Bras 2013;21(5):285-7.

9. Alger J, Becerra-Posada F, Kennedy A, Martinelli E, et al. Sistemas nacionales de investigación para la salud en América Latina: una revisión de 14 países. Rev Panam Salud Publica 2009;26(5):447-57.

10. Rodríguez Pomeda J. Indicadores iberoamericanos de I+D. Revista de Investigación en Gestión de la Innovación y Tecnología 2007;45. Disponibleen: http:/ / www.madrimasd. org/revista/revista45/ImasDCifras/imasdcifras.asp. [Consulta: 6 de febrero de 2014].

11. Red de Indicadores de Ciencia y Tecnología Iberoamericana eInteramericana. Gasto en I+D por sector de financiamiento. Buenos Aires: RICYT;2014. Disponible en: http:/ /db.ricyt. org/query/AR,BO,BR,CA,CL,CO,CR,CU,EC,ES, GT,MX, PA,PT,PY,SV,US,UY,AL,IB/1990\%2C2011/GASIDSFPER. [Consulta: 6 de febrero de 2014].

12. Domínguez P, Chiolo MJ, Davenport MC, Di Lalla S, et al. Evaluación dela producción científica del Hospital General de Niños Pedro de Elizalde, 2000-2011. Arch Argent Pediatr 2014;112(2):147-52.

13. Gregory TN, Liu T, Machuk A, Arneja JS. What is the ultimate fate of presented abstracts? The conversion rates of presentations to publications over a five-year period from three North American plastic surgery meetings. Can J Plast Surg 2012;20(1):33-6.

14. Krzyzanowska MK, Pintilie M, Tannock IF. Factors associated with failure to publish large randomized trials presented at an oncology meeting. JAMA 2003;290(4):495-501.

15. McGrail M, Rickard C, Jones R. Publish or perish: a systematic review of interventions to increase academic publication rates. Higher Education Research \& Development 2006;25:19-35. 
ANEXo. Encuesta utilizada para valorar la publicación de trabajos presentados en reuniones de la Sociedad Latinoamericana de Investigación Pediátrica

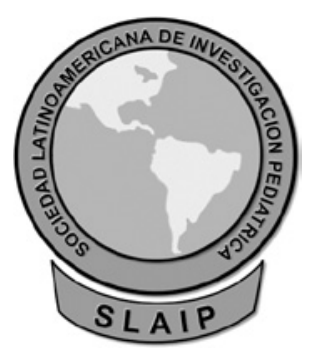

\section{Encuesta}

Evaluación de la publicación científica de trabajos presentados en SLAIP

Estimado colega:

Lo estamos invitando a participar de un estudio sobre la publicación de trabajos científicos presentados en reuniones de la Sociedad Latinoamericana de Investigación Pediátrica. Como Ud. sabrá, a pesar de ser deseable, es limitada la cantidad de trabajos científicos que luego de presentados en congresos alcanzan la publicación en forma completa. Estimar nuestra producción científica y, eventualmente, poder identificar factores que limiten la publicación nos ayudará a diseñar estrategias para encarar esas dificultades. Ud. está siendo contactado porque presentó un trabajo en una reunión de la SLAIP. Su participación en esta encuesta es voluntaria. Sus datos personales serán convenientemente disociados de la información requerida para el estudio de manera que su identidad quedará preservada. El responder la encuesta implicará consentimiento a participar del estudio.

Muchas gracias!

Nombre del trabajo:

Año de presentación en SLAIP:

Por favor, responda las siguientes preguntas marcando con " $\mathrm{X}$ " según corresponda.

1) ¿Su proyecto recibió financiación?

Pública:

Privada:

No:

2) ¿Su proyecto fue publicado en alguna revista científica?

Sí (incluye prensa):

No:

Si su respuesta fue afirmativa indique autores, nombre del estudio, revista, volumen, páginas, año (como cita usualmente una referenciabi bliográfica):

Si su respuesta fue negativa indique cuál considera que fue la razón más importante que impidióla publicación de su investigación (seleccione sólo una).

$\square$ Falta de interés

Falta de tiempo

Falta de acuerdo entre coautores

Otros trabajos con resultados similares u opuestos

Consideró que los resultados obtenidos no eran trascendentes

$\square$ Tamaño muestral insuficiente

Dificultad con el análisis estadístico

Pesimismo de los autores con respecto a la aceptación para su publicación

Dificultad en el financiamiento

No aceptado para su publicación

Otras causas 